\title{
Discrete wavelet transform-based RI adaptive algorithm for system identification
}

\author{
Mohammad Shukri Salman ${ }^{1}$, Alaa Eleyan ${ }^{2}$, Bahaa Al-Sheikh ${ }^{3}$ \\ ${ }^{1,3}$ College of Engineering and Technology, American University of the Middle East, Kuwait \\ ${ }^{2}$ Electrical and Electronic Engineering, Avrasya University, Trabzon, Turkey \\ ${ }^{3}$ Biomedical Systems and Medical Informatics Engineering Department, Yarmouk University, Jordan
}

\begin{abstract}
Article Info
Article history:

Received Mar 20, 2019

Revised Nov 5, 2019

Accepted Nov 25, 2019

Keywords:

Adaptive filters

Discrete wavelet transform

LMS algorithm

Recursive inverse algorithm

System identification

ABSTRACT

In this paper, we propose a new adaptive filtering algorithm for system identification. The algorithm is based on the recursive inverse (RI) adaptive algorithm which suffers from low convergence rates in some applications; i.e., the eigenvalue spread of the autocorrelation matrix is relatively high. The proposed algorithm applies discrete-wavelet transform (DWT) to the input signal which, in turn, helps to overcome the low convergence rate of the RI algorithm with relatively small step-size(s). Different scenarios has been investigated in different noise environments in system identification setting. Experiments demonstrate the advantages of the proposed DWT recursive inverse (DWT-RI) filter in terms of convergence rate and mean-square-error (MSE) compared to the RI, discrete cosine transform LMS (DCT-LMS), discretewavelet transform LMS (DWT-LMS) and recursive-least-squares (RLS) algorithms under same conditions.
\end{abstract}

Copyright (C) 2020 Insitute of Advanced Engineeering and Science. All rights reserved.

\section{Corresponding Author:}

Mohammad Shukri Salman,

College of Engineering and Technology,

American University of the Middle East,

Kuwait.

Tel: +965 2225 1400, ext: 1765

Email: mohammad.salman@aum.edu.kw

\section{INTRODUCTION}

Adaptive filtering plays an essential role in many signal processing applications. The least-meansquare (LMS) algorithm's simplicity made it very commonly used in adaptive signal processing applications such as system identification [1], echo cancelation [2], channel equalization [3, 4], and interference cancelation $[3,5]$. The main drawback of the LMS algorithm is that it suffers from low convergence rate when the input signal is highly correlated. Many LMS variants [6-10] had been introduced to overcome the limitations of the original LMS algorithm and to achieve better performance in terms of mean-square-error (MSE) and convergence rate. Transform domain variable step-size algorithms are effective and performing well in highly correlated noise environment [7, 11]. It has been shown in [12] that transforming the input signal into another domain can reduce the eigenvalue spread of input signal autocorrelation matrix which, in turn, accelerates the convergence rate of the adaptive filters. The discrete-wavelet-transform LMS (DWT-LMS) [10] and the discrete-cosine transform LMS (DCT-LMS) [13] algorithms were proposed to decrease the eigenvalue spread of the autocorrelation matrix of the input signal. However, due to the fixed step-size of the LMS algorithm, these algorithms provide poor performance in terms of the convergence rate.

The recursive-least-square (RLS) algorithm was proposed to offer faster convergence rate, especially, for highly correlated input signals $[6,14,15]$. However, it has a disadvantage of being highly computation- 
ally complex. The performance of RLS algorithm and its variants depends on the forgetting factor $(\beta)$ in terms of convergence rate, misadjustment, tracking capability and stability [16]. The forgetting factor can take values between zero and unity and needs to compromise between the above mentioned performance criteria. When the forgetting factor is close to unity, the algorithm achieves low misadjustment and high stability, but it compromises its tracking capabilities. A smaller value of $(\beta)$ would improve the tracking capability of the RLS algorithm [16], but it would increase the misadjustment and might affect the stability of the algorithm. In [17] the authors investigated the influence of the forgetting factor of the RLS adaptive filter in system identification. A possible solution to overcome this problem is to use a variable forgetting factor (VFF-RLS) algorithm [18, 19].

Recursive inverse (RI) algorithm [20, 21] had been proposed to overcome the drawbacks and limitations of the above mentioned adaptive filters. It had been shown that the RI algorithm performs considerably better than the LMS algorithm and its variants. It was also shown that its performance, in terms of convergence rate and excess MSE, is comparable to that of the RLS under different settings, with less computational complexity. However, still the RI algorithm requires a very small initial value for its step-size if the eigenvalue spread of input signal autocorrelation matrix is relatively high.

In this paper, we introduce a solution for the aforementioned problems by proposing a new DWTbased RI (DWT-RI) algorithm. Applying DWT on the input signal [22, 23] will reduce the eigenvalue spread of the autocorrelation matrix [24, 25] and gives the user the freedom to initiate the RI algorithm with relatively high initial values for the step-size. This process guarantees a very high performance, of the algorithm, in terms of both MSE and convergence rate.

The paper is organized as follows: In Section 2., a brief explanation of discrete wavelet transform is covered. Section 3. presents the derivation of proposed DWT-RI algorithm. In Section 4., simulation results that compare the performance of the proposed algorithm to those of the RI, DCT-LMS, DWT-LMS and RLS algorithms, in different noise environments for system identification setting, are given. Finally, the conclusions are drawn in the last section.

\section{DISCRETE WAVELET TRANSFORM}

The theory of multiresolution analysis was firstly developed by Mallat [26], to represent functions defined over $N$ dimensional space. Wavelet transform is a multiresolution technique for analyzing signals. It was developed, as an alternative to short time Fourier transform (STFT), to overcome the time-frequency resolution problems. The DWT uses filter banks to perform the wavelet analysis by the construction of the multiresolution time-frequency plane. DWT decomposes the signal into wavelet coefficients from which the original signal can be reconstructed again. The wavelet coefficients represent the signal in various frequency bands [27, 28]. Figure 1 shows the structure of discrete wavelet transform adaptive filter (DWTAF).

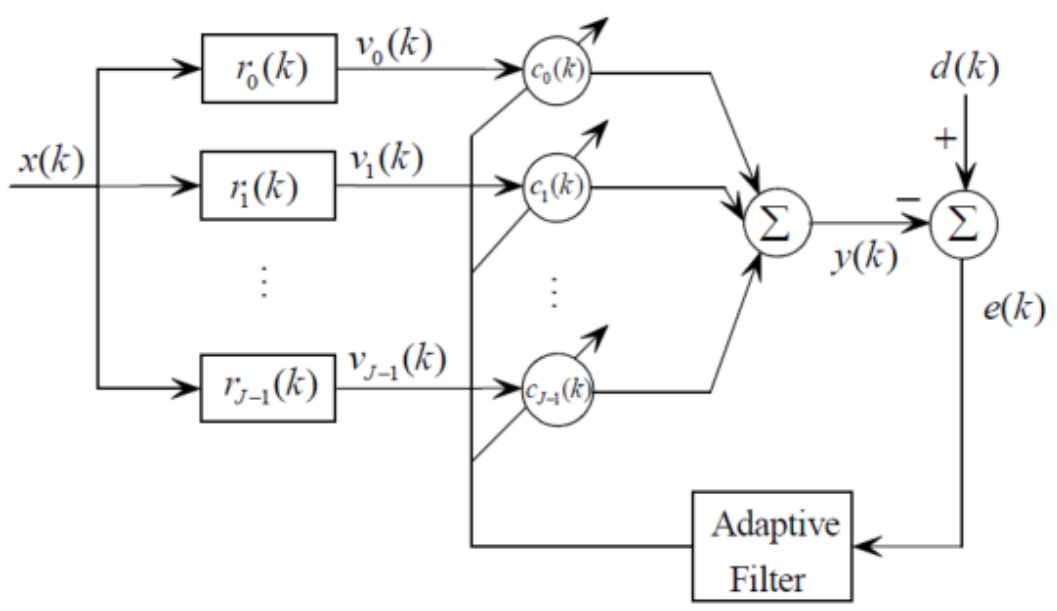

Figure 1. Structure of discrete wavelet transform transversal adaptive filter. 
According to DWT theory, reconstruction of the original signal $\mathbf{x}(k)$ can be performed using the following finite sum:

$$
\mathbf{x}(k)=\sum_{j=0}^{J-1} \sum_{n \in Z} \theta_{j, n} \psi_{j, n}(k),
$$

where $\theta_{j, n}$ are the wavelet coefficients and $\psi_{j, n}(k)$ are the wavelet functions that form an orthogonal basis. The purpose of DWT adaptive filter is to generate the discrete reconstruction of $\mathbf{x}_{j}(k)$ which is the projective discrete form of $\mathbf{x}(k)$ in wavelet subspace. $\mathbf{x}_{j}(k)$ is given by:

$$
\mathbf{x}_{j}(k)=\sum_{n \in Z} \theta_{j, n} \psi_{j, n}(k) .
$$

If $\mathbf{v}_{j}(k)$ is the approximation of projective $\mathbf{x}_{j}(k)$, then

$$
\mathbf{v}_{j}(k)=\sum_{n \in Z} \hat{\theta}_{j, n} \psi_{j, n}(k),
$$

where $\hat{\theta}_{j, n}$ is the discrete approximation of the wavelet coefficients $\theta_{j, n}$,

$$
\hat{\theta}_{j, n}=\sum_{l} \mathbf{x}(l) \bar{\psi}_{j, n}(l)
$$

Given that,

$$
h_{j}(l, k)=\sum_{n \in Z} \bar{\psi}_{j, n}(l) \psi_{j, n}(k) .
$$

Now, substituting (4) and (5) in (3) results in

$$
\mathbf{v}_{j}(k)=\sum_{l} \mathbf{x}(l) h_{j}(l, k) .
$$

Equation (6) is simply the discrete convolution of the input signal $\mathbf{x}(k)$ and the filter coefficients $h_{j}(l, k)$. Using Orthogonality and time-steadiness, filter indices can be rewritten as:

$$
h_{j}(l, k)=h_{j}(l-k) .
$$

Therefore,

$$
\mathbf{v}_{j}(k)=\sum_{l} \mathbf{x}(l) h_{j}(l-k)
$$

\section{DISCRETE WAVELET TRANSFORM RECURSIVE INVERSE ALGORITHM}

\subsection{Recursive inverse (RI) algorithm}

The optimum solution for the FIR filter coefficients can be obtained using the Wiener-Hopf equation [6]:

$$
\mathbf{C}(k)=\mathbf{R}^{-1}(k) \mathbf{p}(k),
$$

where $k$ is the time parameter $(k=1,2, \ldots), \mathbf{C}(k)$ is the filter weight vector calculated at time $k, \mathbf{R}(k)$ is the estimate of the tap-input vector's autocorrelation matrix, and $\mathbf{p}(k)$ is the estimate of the cross-correlation vector between the desired output signal and the tap-input vector.

Recursive estimation of $\mathbf{R}(k)$ and $\mathbf{p}(k)$ in (9) can be obtained as follows;

$$
\begin{gathered}
\mathbf{R}(k)=\beta \mathbf{R}(k-1)+\mathbf{x}(k) \mathbf{x}^{T}(k), \\
\mathbf{p}(k)=\beta \mathbf{p}(k-1)+d(k) \mathbf{x}(k),
\end{gathered}
$$

where $\beta$ is the forgetting factor which is usually very close to unity and $\mathbf{x}(k)$ is the tap-input vector. 
Using the recursive solution of the Wiener-Hopf equation for one iteration with variable step size gives the weight update equation of the RI algorithm [20]:

$$
\mathbf{C}(k)=[\mathbf{I}-\mu(k) \mathbf{R}(k)] \mathbf{C}(k-1)+\mu(k) \mathbf{p}(k) .
$$

Where $\mu(k)$ is the variable step-size [20] which satisfies the convergence criterion [6]:

$$
\mu(k)<\frac{2}{\lambda_{\max }(\mathbf{R}(k))}=\left(\frac{1-\beta}{1-\beta^{k}}\right)\left(\frac{2}{\lambda_{\max }\left(\mathbf{R}_{x x}\right)}\right)=\frac{\mu_{\max }}{1-\beta^{k}},
$$

where $\lambda_{\max }$ is the maximum eigenvalue of $\mathbf{R}(k)$ and $\mathbf{R}_{x x}=E\left\{\mathbf{x}(k) \mathbf{x}^{T}(k)\right\}$. For more details about derivation and convergence analysis of the RI algorithm, the reader may refer to [20].

The RI algorithm has a major advantage compared to the RLS algorithm in that it does not require the update of the inverse autocorrelation matrix. Also, its computational complexity is much less than that of the RLS algorithm. Due to the update of inverse autocorrelation matrix, RLS type algorithms may face numerical stability problems [29], which is not the case for the RI algorithm.

\subsection{The proposed algorithm}

The performance of the RI algorithm can be improved further by applying the DWT to the input signal. In this case, the weights update equation in (12) will become as:

$$
\mathbf{C}(k+1)=\left[\mathbf{I}-\mu(k) \mathbf{R}_{v v}(k)\right] \mathbf{C}(k)+\mu(k) \mathbf{p}_{v d}(k)
$$

where

$$
\mathbf{R}_{v v}(k)=\beta \mathbf{R}_{v v}(k-1)+\mathbf{v}(k) \mathbf{v}^{T}(k),
$$

and

$$
\mathbf{p}_{v d}(k)=\beta \mathbf{p}_{v d}(k-1)+d(k) \mathbf{v}(k) .
$$

Where $\mathbf{v}(k)=\mathbf{W} \mathbf{x}(k)$ is the transformed input signal and $\mathbf{W}$ is the wavelet transform matrix of size $J \times N$.

$$
\mu(k)=\frac{\mu_{0}}{1-\beta^{k}}
$$

where $\mu_{0}$ is a constant selected as:

$$
\mu_{0}<\mu_{\max }=\frac{2(1-\beta)}{\lambda_{\max }\left(\mathbf{R}_{v v}\right)} \quad \text { and } \quad \mathbf{R}_{v v}=E\left[\mathbf{v}(k) \mathbf{v}^{T}(k)\right]
$$

The adaptive estimation error is given as:

$$
\mathbf{e}(k)=\mathbf{d}(k)-\mathbf{y}(k)
$$

where

$$
\mathbf{y}(k)=\mathbf{v}^{T}(k) \mathbf{C}(k)=\sum_{j=0}^{J-1} v_{j}(k) c_{j}(k)=\sum_{j=0}^{J-1} \sum_{l} c_{j}(k) h_{j}(l-k) x(l),
$$

where $\mathbf{y}(k)$ is the transformed version of $y(k)$ shown in Figure 2 


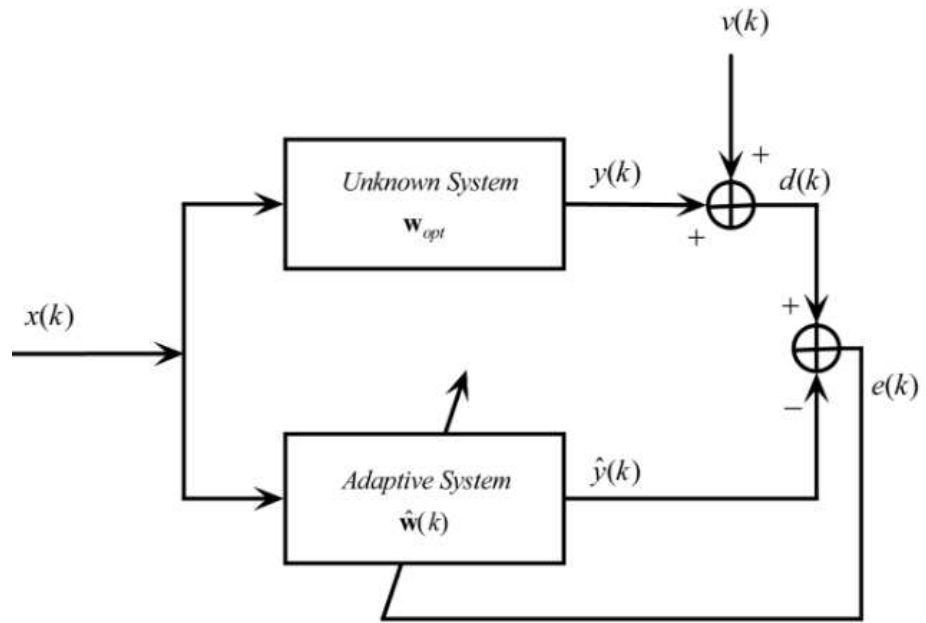

Figure 2. Block diagram of an adaptive system identification setting

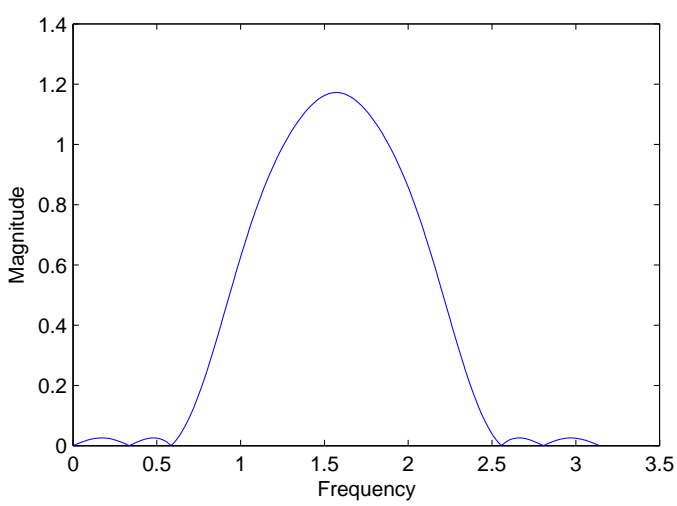

Figure 3. Unknown system transfer function

\section{SIMULATION RESULTS}

In the simulations, the performance of the proposed DWT-RI algorithm is compared to those of the RI [20], DCT-LMS [9], DWT-LMS [10] and RLS [6] algorithms in the system identification setting described in Figure 2. The filter length for all algorithms is $J=16$ taps and the signal-to-noise ratio (SNR) was selected to be $30 \mathrm{~dB}$ for all the experiments. The received signal $\mathbf{x}(k)$ was generated using the DWT on the signal $x_{i}(k)$ given by [7]:

$$
x_{i}(k)=1.79 x_{i}(k-1)-1.85 x_{i}(k-2)+1.27 x_{i}(k-3)-0.41 x_{i}(k-4)+v_{0}(k) .
$$

where $v_{0}(k)$ is a Gaussian process with zero mean and variance $\sigma^{2}=0.3849$. The unknown system is the bandpass filter shown in Figure 3. The simulation results for Gaussian and impulsive noise were obtained by averaging 100 and 300 Monte-Carlo independent runs, respectively.

\subsection{Additive white gaussian noise}

In order to test the performance of the proposed algorithm, the signal is assumed to be corrupted with an additive white Gaussian noise (AWGN) process. Simulations were carried out with the following parameters: For the DWT-RI and RI algorithms: $\beta=0.99$ and $\mu_{0}=0.0013$. For DCT-LMS algorithm: $\beta=0.9985$, $\epsilon=8 \times 10^{-4}, \mu=0.02, \gamma=2 \times 10^{-3}$ and $M=10$. For DWT-LMS algorithm: $\mu=0.02$. For the RLS algorithm: $\beta=0.99$. Figure 4 shows that the RI, RLS and DWT-RI algorithms converge to the same 
MSE. However, the DWT-RI and RLS converge much faster than the RI algorithm (950 iterations faster). On the other hand, the DCT-LMS and DWT-LMS algorithms converge to a higher MSE (mse $=5 \mathrm{~dB}$ worse) than the other algorithms with low rate of convergence.

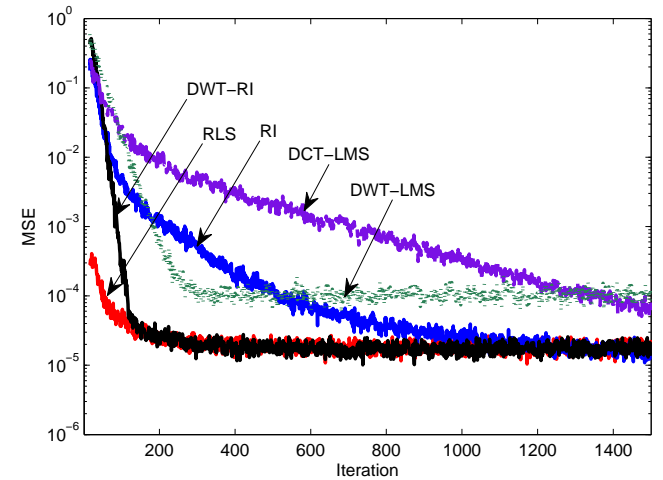

Figure 4. The ensemble MSE for RI, RLS, DCT-LMS, DWT-LMS and DWT-RI in AWGN

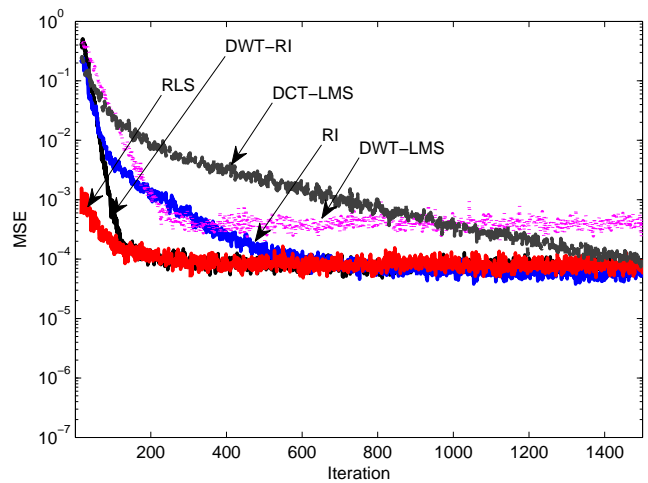

Figure 5. The ensemble MSE for RI, RLS, DCT-LMS, DWT-LMS and DWT-RI in AWIN

$$
(\epsilon=0.2, \kappa=100)
$$

\subsection{Additive white impulsive noise}

Due to man-made noise, underwater acoustic noise, atmospheric noise, etc, the noise added to the received signal may not be modeled as Gaussian. This type of noise which has a heavy-tailed distribution is characterized by outliers and may be better modeled using a Gaussian mixture model. In order to test the robustness of the proposed algorithm, and to study the effects of the impulsive components (outliers) of the noise process in the system identification setting, an impulsive noise process is generated by the probability density function [30]: $f=(1-\epsilon) G\left(0, \sigma_{n}^{2}\right)+\epsilon G\left(0, \kappa \sigma_{n}^{2}\right)$ with variance $\sigma_{f}^{2}$ given as: $\sigma_{f}^{2}=(1-\epsilon) \sigma_{n}^{2}+\epsilon \kappa \sigma_{n}^{2}$, where $G\left(0, \sigma_{n}^{2}\right)$ is a Gaussian probability density function with zero mean and variance $\sigma_{n}^{2}$ that represents the nominal background noise. $G\left(0, \kappa \sigma_{n}^{2}\right)$ represents the impulsive component of the noise model, where $\epsilon$ is the probability and $\kappa \geq 1$ is the strength of the impulsive components.

(a) Firstly, the signal is assumed to be corrupted with an additive white impulsive noise (AWIN) process. The white impulsive noise process is generated with the parameters: $\epsilon=0.2$ and $\kappa=100$. Simulations were carried out with the following parameters: For the DWT-RI and RI algorithms: $\beta=0.99$ and $\mu_{0}=0.001$. For DCT-LMS algorithm: $\beta=0.9985, \epsilon=8 \times 10^{-4}, \mu=0.02, \gamma=2 \times 10^{-3}$ and $M=10$. For DWT-LMS algorithm: $\mu=0.018$. For the RLS algorithm: $\beta=0.99$. Figure 5 shows that the DWT-RI and RLS algorithms converge to the same MSE at the same time, The RI and DCT-LMS algorithms still converge to the same MSE but with much low rate of convergence than those of the DWT-RI and RLS algorithms. The DWT-LMS algorithm converges to much higher MSE with almost the same rate of convergence of those of RLS and DWT-RI algorithms.

(b) Secondly, in order to emphasize on the capability of the proposed DWT-RI algorithm in suppressing impulsive noise, even, with high impulsivity strength, an AWIN process is generated with the parameters: $\epsilon=0.2$ and $\kappa=100000$. Simulations were carried out with the following parameters: For the DWT-RI and RI algorithms: $\beta=0.99$ and $\mu_{0}=0.0001$. For DCT-LMS algorithm: $\beta=0.9985$, $\epsilon=8 \times 10^{-4}, \mu=0.02, \gamma=2 \times 10^{-3}$ and $M=10$. For DWT-LMS algorithm: $\mu=0.018$. For the RLS algorithm: $\beta=0.99$. The RI algorithm fails to converge under these settings. Figure 6 shows that the RLS algorithm converges to the steady-state MSE (MSE $=-10 \mathrm{~dB}$ ) after 400 time iterations, while the DWT-RI algorithm converges to a lower MSE (MSE $=-14 \mathrm{~dB}$ ) than the RLS at the same time. The DCT-LMS algorithm converges to a lower MSE than that of the DWT-RI algorithm but with very low convergence rate, whereas the DWT-LMS algorithm converges to a very high MSE. This shows the advantage of domain transform in reducing the self-correlation of the input signal and converging to a lower MSE values with higher convergence rates. 


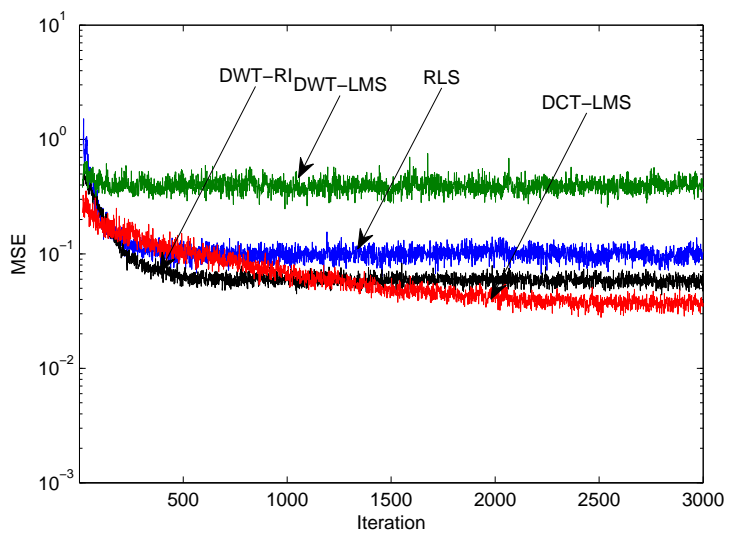

Figure 6. The ensemble MSE for RI, RLS, DCT-LMS, DWT-LMS and DWT-RI in AWIN

$$
(\epsilon=0.2, \kappa=100000)
$$

\section{CONCLUSION}

A new discrete wavelet transform based RI adaptive filtering algorithm is proposed. The performances of the DWT-RI, DCT-LMS, DWT,LMS, RI and RLS algorithms are compared in AWGN and AWIN environments in system identification setting. Under the same conditions, the DWT-RI algorithm outperforms the aforementioned algorithms in terms of MSE and/or convergence rate.

\section{REFERENCES}

[1] M. S. Ahmad, O. Kukrer and A. Hocanin, "The effect of the forgetting factor on the RI adaptive algorithm in system identification," 10th International Symposium on Signals, Circuits and Systems (ISSCS), pp. 14, 2011.

[2] C. Paleologu, J. Benesty, S. L. Grant, and C. Osterwise, "Variable step-size NLMS algorithms for echo cancellation," 43rd Asilomar Conference on Signals, Systems and Computers, pp. 633-637, 2009.

[3] C. V. Sinn, and J. Gotze, "Comparative study of techniques to compute FIR filter weights in adaptive channel equalization," IEEE International Conference on Acoustics, Speech and Signal Processing (ICASSP03), vol. 6, pp. 217-220, 2003.

[4] M. S. Ahmad, O. Kukrer and A. Hocanin, "An efficient Recursive Inverse adaptive filtering algorithm for channel equalization," European Wireless Conference (EW 2010), pp. 88-92, 2010.

[5] S. Dixit and D. Nagaria, "LMS adaptive filters for noise cancellation: A review," International Journal of Electrical and Computer Engineering (IJECE), vol. 7, no. 5, pp. 2520-2529, 2017.

[6] S. Haykin, Adaptive Filter Theory, Prentice Hall, Upper Saddle River, NJ, $4^{\text {th }}$ edn., 2002.

[7] R. C. Bilcu, P. Kuosmanen and K. Egiazarian, "A transform domain LMS adaptive filter with variable step-size," IEEE Signal Processing Letters, vol. 9, no. 2, pp. 51-53, 2002.

[8] C. P. Kwong and E. W. Jonston, "A variable step-size LMS algorithm," IEEE Transactions on Signal Processing, vol. 40, pp. 1633-1642, 1992.

[9] D. I. Kim and P. De Wilde, "Performance analysis of the DCT-LMS adaptive filtering algorithm," Signal Process, vol. 80, no. 8, pp. 1629-1654, 2000.

[10] S. Hosur and A. H. Tewfik, "Wavelet transform domain adaptive FIR filtering," IEEE Transactions on Signal Processing, vol. 45, no. 3, pp. 617-629, 1997.

[11] T. Gowri, R. Kumar P., D.V.R. Koti Reddy, "Efficient reduction of PLI in ECG signal using new variable step size least mean fourth adaptive algorithm," International Journal of Electrical and Computer Engineering (IJECE), vol. 9, no. 1, pp. 307-313, 2019.

[12] S. S. Narayan, A. M. Peterson and M. J. Narasimha, "Transform domain LMS algorithm," IEEE Transactions on ASSP, ASSP-31, no. 3, pp. 609-615, 1983.

[13] J. C. Lee and C. K. Un, "Performance of transform domain LMS adaptive digital filters," IEEE Transactions of Acoustic, Speech, Signal Processing, ASSP-34, no. 3, pp. 499-510, 1986. 
[14] S. Qiao, "Fast adaptive RLS algorithms: a generalized inverse approach and analysis," IEEE Transactions on Signal Processing, vol. 39, no. 6, pp. 1455-1459, 1991.

[15] A. H. Sayed, Adaptive Filters, John Wily \& Sons, NJ, 2008.

[16] S. Haykin, A. H. Sayed, J. R. Zeidler, P. Yee and P. C. Wei, "Adaptive tracking of linear time-variant systems by extended RLS algorithms," IEEE Transactions on Signal Processing, vol. 45, no. 5, pp. 11181128, 1997.

[17] S. Ciochina, C. Paleologu, J. Benesty and A. A. Enescu, "On the influence of the forgetting factor of the RLS adaptive filter in system identification," International Symposium on Signals, Circuits and Systems (ISSCS 2009), pp. 1-4, 2009.

[18] S. H. Leung, and C. F. So, "Gradient-based variable forgetting factor RLS algorithm in time-varying environments," IEEE Transactions on Signal Processing, vol. 53, no. 8, pp. 3141-3150, 2005.

[19] C. Paleologu, J. Benesty and S. Ciochina, "A robust variable forgetting factor recursive least-squares algorithm for system identification," IEEE Signal Processing Letters, vol. 15, pp. 597-600, 2008.

[20] M. S. Ahmad, O. Kukrer and A. Hocanin, "Recursive inverse adaptive filtering algorithm," Digital Signal Processing (Elsevier), vol. 21, no. 4, pp. 491-496, 2011.

[21] M. S. Salman, O. Kukrer and A. Hocanin, "Recursive inverse algorithm: Mean-square-error analysis," Digital Signal Processing (Elsevier), vol. 66, pp. 10-17, 2017.

[22] S. Saxena, R. Jais and M. K. Hota, "Removal of powerline interference from ECG signal using FIR, IIR, DWT and NLMS adaptive filter," 2019 International Conference on Communication and Signal Processing (ICCSP), pp. 12-16, 2019.

[23] A. Mannan and A. Habib, "Adaptive processing of image using DWT and FFT OFDM in AWGN and Rayleigh channel," 2017 International Conference on Communication, Computing and Digital Systems (C-CODE), pp. 346-350, 2017.

[24] T. Yang, Y. Chiang and F. Chang, "Further discussions on adaptive filters to improve the wavelet shrinkage method," 2016 International Conference on System Science and Engineering (ICSSE), pp. 1-4, 2016.

[25] A. N. S. Belgurzi and I. Elshafiey, "A power line interference canceler using wavelet transform and adaptive filter for ECG signal," 2017 International Conference on Computer and Applications (ICCA), pp. 206-210, 2017.

[26] S. Mallat, "Wavelet for a vision," Proceedings of the IEEE, vol. 84, no. 4, pp. 604-914, 1996.

[27] A. N. S. Belgurzi and I. Elshafiey, "A power line interference canceler using wavelet transform and adaptive filter for ECG signal," 2017 International Conference on Computer and Applications (ICCA), pp. 206-210, 2017.

[28] A. Mannan and A. Habib, "Adaptive processing of image using DWT and FFT OFDM in AWGN and Rayleigh channel," 2017 International Conference on Communication, Computing and Digital Systems (C-CODE), pp. 346-350, 2017.

[29] G. O. Glentis, K. Berberidis and S. Theodoridis, "Efficient least squares adaptive algorithms for FIR transversal filtering," IEEE Signal Processing Magazine, pp. 13-41, 1999.

[30] H. Delic and A. Hocanin, "Robust detection in DS/CDMA," IEEE Transactions on Vehicular Technology, vol. 51, no. 1, pp. 155-170, 2002.

\section{BIOGRAPHIES OF AUTHORS}

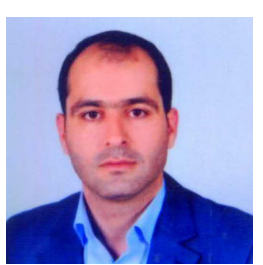

Mohammad Shukri Salman received the B.Sc., M.Sc. and Ph.D. Degrees in Electrical and Electronics Engineering from Eastern Mediterranean University (EMU), in 2006, 2007 and 2011, respectively. From 2006 to 2010, he was a teaching assistant of Electrical and Electronics Engineering department at EMU. In 2010, he has joined the Department of Electrical and Electronic Engineering at European University of Lefke (EUL) as a senior lecturer. For the period 2011-2015, he has worked as an Assist. Prof. in the Department of Electrical and Electronics Engineering, Mevlana (Rumi) University, Turkey. Currently, he is with Electrical Engineering Department at the American University of Middle East in Kuwait. He has served as a general chair, program chair and a TPC member for many international conferences. His research interests include signal processing, adaptive filters, image processing, sparse representation of signals, control systems and communications systems. 


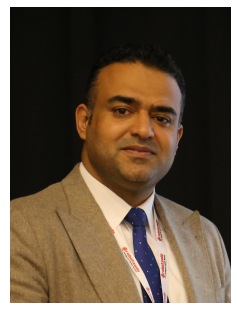

Alaa Eleyan received the B.Sc. and M.Sc. degrees in Electrical Electronics Engineering from Near East University, Northern Cyprus, in 2002 and 2004, respectively. In 2009, He finished his PhD degree in Electrical and Electronics Engineering from Eastern Mediterranean University, Northern Cyprus. Currently, he is working as an associate professor at Avrasya University, Turkey. His current research interests are computer vision, signal image processing, machine learning and robotics. He has more than 60 published journal articles and conference papers in these research fields.

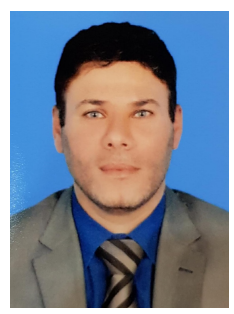

Bahaa Al-Sheikh received the B.Sc. degree in Electronics Engineering from Yarmouk University, Jordan, MSc in Electrical Engineering from Colorado State University, Colorado, USA, and PhD in Biomedical Engineering degree from the University of Denver, Colorado, USA, in 2000, 2005 and 2009, respectively. Between 2009 and 2015, he worked for Yarmouk University as an assistant professor in the department of Biomedical Systems and Medical Informatics Engineering and served as the department chairman between 2010 and 2012. He served as a part-time consultant for Sandhill Scientific Inc., Highlands Ranch, Colorado, USA in Biomedical Signal Processing field between 2009 and 2014. Currently, he is an Assistant Professor at the Electrical Engineering Department at the American University of the Middle East in Kuwait. His research interests include digital signal and image processing, biomedical systems modeling, medical instrumentation and sound source localization systems. 